\title{
A soft set theoretic approach to an AG-groupoid via ideal theory with applications
}

\author{
Faisal Yousafzai ${ }^{1}$ and Mohammed M. Khalaf $2^{2^{*}}$
}

\author{
${ }^{*}$ Correspondence: \\ khalfmohammed2003@yahoo.com \\ ${ }^{2}$ Higher Institute of Engineering \\ and Technology, King Mariout, \\ Alexandria, Egypt \\ Full list of author information is \\ available at the end of the article
}

\begin{abstract}
In this paper, we study the structural properties of a non-associative algebraic structure called an AG-groupoid by using soft set theory. We characterize a right regular class of an AG-groupoid in terms of soft intersection ideals and provide counter examples to discuss the converse part of various problems. We also characterize a weakly regular class of an $\mathrm{AG}^{* *^{*}}$-groupoid by using generated ideals and soft intersection ideals. We investigate the relationship between si-left-ideal, sl-right-ideal, sı-two-sided-ideal, and sl-interior-ideal of an AG-groupoid over a universe set by providing some practical examples.
\end{abstract}

Keywords: Left invertive law, Soft-sets, AG-groupoid, Right regularity, Weak regularity and sl-ideals

\section{Introduction}

The concept of soft set theory was introduced by Molodtsov in [16]. This theory can be used as a generic mathematical tool for dealing with uncertainties. In soft set theory, the problem of setting the membership function does not arise, which makes the theory easily applied to many different fields [1, 2, 5-9]. At present, the research work on soft set theory in algebraic fields is progressing rapidly [19, 21-23] . A soft set is a parameterized family of subsets of the universe set. In the real world, the parameters of this family arise from the view point of fuzzy set theory. Most of the researchers of algebraic structures have worked on the fuzzy aspect of soft sets. Soft set theory is applied in the field of optimization by Kovkov in [12]. Several similarity measures have been discussed in [15], decision-making problems have been studied in [21], and reduction of fuzzy soft sets and its applications in decision-making problems have been analyzed in [13]. The notions of soft numbers, soft derivatives, soft integrals, and many more have been formulated in [14]. This concept have been used for forecasting the export and import volumes in international trade [28]. A. Sezgin have introduced the concept of a soft sets in non-associative semigroups in [24] and studied soft intersection left (right, two-sided) ideals, (generalized) bi-ideals, interior ideals, and quasi-ideals in an AG-groupoid. A lot of work has been done on the applications of soft sets to a non-associative rings by T. Shah et al. in $[25,26]$. They have characterized the non-associative rings through soft M-systems and different soft ideals to get generalized results.

(c) The Author(s). 2019 Open Access This article is distributed under the terms of the Creative Commons Attribution 4.0 International License (http://creativecommons.org/licenses/by/4.0/), which permits unrestricted use, distribution, and reproduction in any medium, provided you give appropriate credit to the original author(s) and the source, provide a link to the Creative Commons license, and indicate if changes were made. 
This paper is the continuation of the work carried out by F. Yousafzai et al. in [29] in which they define the smallest one-sided ideals in an AG-groupoid and use them to characterize a strongly regular class of an AG-groupoid along with its semilattices and soft intersection left (right, two-sided) ideals, and bi-ideals. The main motivation behind this paper is to study some structural properties of a non-associative structure as it has not attracted much attention compared to associative structures. We investigate the notions of SI-left-ideal, sI -right-ideal, sI-two-sided-ideal, and sI -interior-ideal in an AG-groupoid. We provide examples/counter examples for these sI-ideals and study the relationship between them in detail. As an application of our results, we get characterizations of a right regular AG-groupoid and weakly regular $\mathrm{AG}^{* * * *}$-groupoid in terms of si-left-ideal, SI-right-ideal, sI-two-sided-ideal, and sI-interior-ideal.

\section{AG-groupoids}

An AG-groupoid is a non-associative and a non-commutative algebraic structure lying in a gray area between a groupoid and a commutative semigroup. Commutative law is given by $a b c=c b a$ in ternary operations. By putting brackets on the left of this equation, i.e., $(a b) c=(c b) a$, in 1972, M. A. Kazim and M. Naseeruddin introduced a new algebraic structure called a left almost semigroup abbreviated as an LA-semigroup [10]. This identity is called the left invertive law. P. V. Protic and N. Stevanovic called the same structure an Abel-Grassmann's groupoid abbreviated as an AG-groupoid [20].

This structure is closely related to a commutative semigroup because a commutative AG-groupoid is a semigroup [17]. It was proved in [10] that an AG-groupoid $S$ is medial, that is, $a b \cdot c d=a c \cdot b d$ holds for all $a, b, c, d \in S$. An AG-groupoid may or may not contain a left identity. The left identity of an AG-groupoid permits the inverses of elements in the structure. If an AG-groupoid contains a left identity, then this left identity is unique [17]. In an AG-groupoid $S$ with left identity, the paramedial law $a b \cdot c d=d c \cdot b a$ holds for all $a, b, c, d \in S$. By using medial law with left identity, we get $a \cdot b c=b \cdot a c$ for all $a, b, c \in S$. We should genuinely acknowledge that much of the ground work has been done by M. A. Kazim, M. Naseeruddin, Q. Mushtaq, M. S. Kamran, P. V. Protic, N. Stevanovic, M. Khan, W. A. Dudek, and R. S. Gigon. One can be referred to [3, 4, 11, 17, 18, 20, 27] in this regard.

A nonempty subset $A$ of an AG-groupoid $S$ is called a left (right, interior) ideal of $S$ if $S A \subseteq A$ ( $A S \subseteq A, S A \cdot S \subseteq A$ ). Equivalently, a nonempty subset $A$ of an AG-groupoid $S$ is called a left (right, interior) ideal of $S$ if $S A \subseteq A$ ( $A S \subseteq A, S A \cdot S \subseteq A$ ). By two-sided ideal or simply ideal, we mean a nonempty subset of an AG-groupoid $S$ which is both left and right ideal of $S$.

\section{Soft sets}

In [23], Sezgin and Atagun introduced some new operations on soft set theory and defined soft sets in the following way:

Let $U$ be an initial universe set, $E$ a set of parameters, $P(U)$ the power set of $U$, and $A \subseteq E$. Then, a soft set (briefly, a soft set) $f_{A}$ over $U$ is a function defined by:

$$
f_{A}: E \rightarrow P(U) \text { such that } f_{A}(x)=\emptyset \text {, if } x \notin A \text {. }
$$

Here, $f_{A}$ is called an approximate function. A soft set over $U$ can be represented by the set of ordered pairs as follows: 


$$
f_{A}=\left\{\left(x, f_{A}(x)\right): x \in E, f_{A}(x) \in P(U)\right\} .
$$

It is clear that a soft set is a parameterized family of subsets of $U$. The set of all soft sets is denoted by $S(U)$.

Let $f_{A}, f_{B} \in S(U)$. Then, $f_{A}$ is a soft subset of $f_{B}$, denoted by $f_{A} \simeq f_{B}$ if $f_{A}(x) \subseteq f_{B}(x)$ for

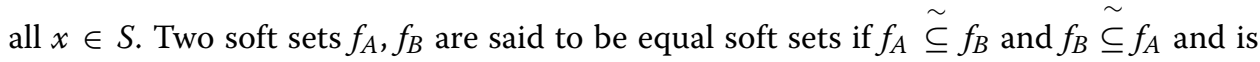

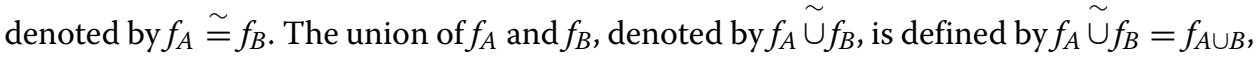
where $f_{A \cup B}(x)=f_{A}(x) \cup f_{B}(x), \forall x \in E$. In a similar way, we can define the intersection of $f_{A}$ and $f_{B}$.

Let $f_{A}, f_{B} \in S(U)$. Then, the soft product [23] of $f_{A}$ and $f_{B}$, denoted by $f_{A} \circ f_{B}$, is defined as follows:

$$
\left(f_{A} \circ f_{B}\right)(x)=\left\{\begin{array}{cc}
\bigcup_{x=y z}\left\{f_{A}(y) \cap g_{B}(z)\right\} & \text { if } \exists y, z \in S \ni x=y z \\
\varnothing & \text { otherwise }
\end{array} .\right.
$$

Let $f_{A}$ be a soft set of an AG-groupoid $S$ over a universe $U$. Then, $f_{A}$ is called a soft intersection left ideal, right ideal, interior ideal (briefly, SI -left-ideal, sI-right-ideal, SIinterior-ideal) of $S$ over $U$ if it satisfies $f_{A}(x y) \supseteq f_{A}(y)\left(f_{A}(x y) \supseteq f_{A}(x), f_{A}(x y \cdot z) \supseteq f_{A}(y)\right)$, $\forall x, y \in S$. A soft set $f_{A}$ is called a soft intersection two-sided ideal (briefly, SI -two-sidedideal) of $S$ over $U$ if $f_{A}$ is an sI -left-ideal and an si-right-ideal of $S$ over $U$.

Let $A$ be a nonempty subset of $S$. We denote by $X_{A}$ the soft characteristic function of $A$ and define it as follows:

$$
X_{A}=\left\{\begin{array}{l}
U \text { if } x \in A \\
\emptyset \text { if } x \notin A
\end{array} .\right.
$$

Note that the soft characteristic mapping of the whole set $S$, denoted by $X_{S}$, is called an identity soft mapping.

\section{Basic results}

Lemma 1 [29] For a nonempty subset A of an AG-groupoid S, the following conditions are equivalent:

(i) A is a left ideal (right ideal, interior ideal) of $S$;

(ii) A soft set $X_{A}$ of $S$ over $U$ is an sI-left-ideal (sI-right-ideal, st-interior-ideal) of $S$ over $U$.

Lemma 2 [29] Let $S$ be an AG-groupoid. For $\emptyset \neq A, B \subseteq S$, the following assertions hold:

(i) $X_{A} \tilde{\cap} X_{B}=X_{A \cap B}$;

(ii) $X_{A} \circ X_{B}=X_{A B}$.

Remark 1 [29] The set $(S(U)$, o) forms an AG-groupoid and satisfies all the basic laws.

Remark 2 [29] If $S$ is an AG-groupoid, then $X_{S} \circ X_{S}=X_{S}$.

Lemma 3 Let $f_{A}$ be any soft set of a right regular AG-groupoid $S$ with left identity over $U$. Then, $f_{A}$ is an sI-right-ideal (sI-left-ideal, sI-interior-ideal) of $S$ over $U$ if and only if $f_{A}=f_{A} \circ X_{S}\left(f_{A}=X_{S} \circ f_{A}, f_{A}=\left(X_{S} \circ f_{A}\right) \circ X_{S}\right)$ and $f_{A}$ is soft semiprime. 
Proof It is simple.

Lemma 4 For every SI-interior-ideal $f_{A}$ of a right regular AG-groupoid $S$ with left identity over $U, f_{A}=X_{S} \circ f_{A}=f \circ X_{S}$.

Proof Assume that $f_{A}$ is any sI-interior-ideal of $S$ with left identity over $U$. Then, by using Remark 2 and Lemma 3, we have $X_{S} \circ f_{A}=\left(X_{S} \circ X_{S}\right) \circ f_{A}=\left(f_{A} \circ X_{S}\right) \circ X_{S}=$ $\left(f_{A} \circ X_{S}\right) \circ\left(X_{S} \circ X_{S}\right)=\left(X_{S} \circ X_{S}\right) \circ\left(X_{S} \circ f_{A}\right)=\left(\left(X_{S} \circ f_{A}\right) \circ X_{S}\right) \circ X_{S}=f_{A} \circ X_{S}$ and $X_{S} \circ f_{A}=\left(X_{S} \circ X_{S}\right) \circ f_{A}=\left(f_{A} \circ X_{S}\right) \circ X_{S}=\left(X_{S} \circ f_{A}\right) \circ X_{S}=f_{A}$.

Lemma 5 [29] $L e t f_{A}$ be any soft set of an AG-groupoid $S$ over $U$. Then, $f_{A}$ is an SI-rightideal ( SI-left-ideal) of $S$ over $U$ if and only iff $f_{A} \circ X_{S} \subseteq f_{A}\left(X_{S} \circ f_{A} \subseteq f_{A}\right)$.

Lemma 6 A right (left, two-sided) ideal $R$ of an AG-groupoid $S$ is semiprime if and only if $X_{R}$ is soft semiprime over $U$.

Proof Let $R$ be a right ideal of $S$. By Lemma $1, X_{R}$ is an sI-right-ideal of $S$ over $U$. If $a \in S$, then by given assumption $\left(X_{R}\right)(a) \supseteq\left(X_{R}\right)\left(a^{2}\right)$. Now $a^{2} \in R$, implies that $a \in R$. Thus every right ideal of $S$ is semiprime. The converse is simple. Similarly every left or two-sided ideal of $S$ is semiprime if and only if its identity soft mapping is soft semiprime over $U$.

Corollary 1 If any sI-right-ideal (SI-left-ideal, SI-two-sided-ideal) of an AG-groupoid S is $S$-semiprime, then any right (left, two-sided) ideal of $S$ is semiprime.

The converse of Lemma 6 is not true in general which can be followed from the following example.

Example 1 Let us consider an initial universe set $U$ given by $U=\mathbb{Z}$, and $S=\{1,2,3,4,5\}$ be a set of parameters with the following binary operation.

\begin{tabular}{l|lllll}
$*$ & 1 & 2 & 3 & 4 & 5 \\
\hline 1 & 1 & 1 & 1 & 1 & 1 \\
2 & 1 & 5 & 5 & 3 & 5 \\
3 & 1 & 5 & 5 & 2 & 5 \\
4 & 1 & 2 & 3 & 4 & 5 \\
5 & 1 & 5 & 5 & 5 & 5
\end{tabular}

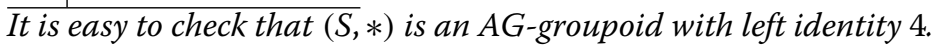

Notice that the only left ideals of $S$ are $\{1,2,5\},\{1,3,5\},\{1,2,3,5\}$ and $\{1,5\}$ respectively which are semiprime. Clearly, the right and two-sided ideals of $S$ are $\{1,2,3,5\}$ and $\{1,5\}$ which are also semiprime. On the other hand, let $A=S$ and define a soft set $f_{A}$ of $S$ over $U$ as follows:

$$
f_{A}(x)=\left\{\begin{array}{c}
\mathbb{Z} \text { if } x=1 \\
4 \mathbb{Z} \text { if } x=2 \\
4 \mathbb{Z} \text { if } x=3 \\
8 \mathbb{Z} \text { if } x=4 \\
2 \mathbb{Z} \text { if } x=5
\end{array}\right\} .
$$


Then, $f_{A}$ is an SI-right-ideal (SI-left-ideal, sI-two-sided-ideal) of $S$ over $U$ but $f_{A}$ is not soft semiprime. Indeed $f_{A}(2) \nsupseteq f_{A}\left(2^{2}\right)$.

Remark 3 If any SI-interior-ideal of an AG-groupoid S with left identity over $U$ is an Ssemiprime over $U$, then any interior ideal of $S$ is semiprime. The converse inclusion is not true in general.

The following lemma will be used frequently in upcoming section without mention in the sequel.

Lemma 7 Let $S$ be an AG-groupoid with left identity. Then, Sa and $S a^{2}$ are the left and interior ideals of $S$ respectively.

Proof It is simple.

\section{Right regular AG-groupoids}

An element $a$ of an AG-groupoid $S$ is called a left (right) regular element of $S$ if there exists some $x \in S$ such that $a=a^{2} x\left(a=x a^{2}\right)$ and $S$ is called left (right) regular if every element of $S$ is left (right) regular.

Remark 4 Let $S$ be an AG-groupoid with left identity. Then, the concepts of left and right regularity coincide in $S$.

Indeed, for every $a \in S$ there exist some $x, y \in S$ such that $a=x a^{2}=a^{2} y$. As $a=x a^{2}=$ $e x \cdot a a=a a \cdot x e=a^{2} y$, and $a=a^{2} y=x a^{2}$ also holds in a similar way.

Let us give an example of an AG-groupoid which will be used for the converse parts of various problems in this section.

Example 2 Let us consider an AG-groupoid $S=\{1,2,3,4,5\}$ with left identity 4 defined in the following multiplication table.

\begin{tabular}{l|lllll}
$*$ & 1 & 2 & 3 & 4 & 5 \\
\hline 1 & 1 & 1 & 1 & 1 & 1 \\
2 & 1 & 5 & 5 & 3 & 5 \\
3 & 1 & 5 & 5 & 2 & 5 \\
4 & 1 & 2 & 3 & 4 & 5 \\
5 & 1 & 5 & 5 & 5 & 5
\end{tabular}

It is easy to check that $S$ is non-commutative and non-associative.

An AG-groupoid $S$ is called left (right) duo if every left (right) ideal of $S$ is a two-sided ideal of $S$ and is called duo if it is both left and right duo. Similarly an AG-groupoid $S$ is called an SI-left (sI-right) duo if every si-left-ideal ( sI-right-ideal) of $S$ over $U$ is an sI-twosided-ideal of $S$ over $U$, and $S$ is called an si-duo if it is both an sI-left and an SI-right duo.

Lemma 8 If every sI-left-ideal of an AG-groupoid $S$ with left identity over $U$ is an SIinterior-ideal of $S$ over $U$, then $S$ is left duo.

Proof Let $I$ be any left ideal of $S$ with left identity. Now by Lemma 1, the identity soft mapping $X_{I}$ is an sI-left-ideal of $S$ over $U$. Thus, by hypothesis, $X_{I}$ is an si-interior-ideal 
of $S$ over $U$, and by using Lemma 1 again, $I$ is an interior ideal of $S$. Thus $I S=I \cdot S S=$ $S \cdot I S=S S \cdot I S=S I \cdot S S=S I \cdot S \subseteq I$. This shows that $S$ is left duo.

The converse part of Lemma 8 is not true in general. Let us consider an AG-groupoid $S$ (from Example 2). It is easy to see that $S$ is left duo because the only left ideal of $S$ is $\{1,5\}$ which is also a right ideal of $S$. Let $A=S$ and define a soft set $f_{A}$ of $S$ over $U=\left\{p_{1}, p_{2}, p_{3}, p_{4,} p_{5}, p_{6}\right\}$ as follows:

$$
f_{A}(x)=\left\{\begin{array}{c}
U \text { if } x=1 \\
\left\{p_{1}, p_{2}, p_{3}, p_{4}\right\} \text { if } x=2 \\
\left\{p_{2}, p_{3}, p_{4}, p_{5}\right\} \text { if } x=3 \\
\left\{p_{3}, p_{4}, p_{5}\right\} \text { if } x=4 \\
\left\{p_{1}, p_{2}, p_{3}, p_{4,} p_{5}\right\} \text { if } x=5
\end{array}\right\} .
$$

Then, it is easy to see that $f_{A}$ is an SI -left-ideal of $S$ over $U$ but it is not an SI -interior-ideal of $S$ over $U$ because $f_{A}(42 * 4) \nsupseteq f_{A}(2)$.

Corollary 2 Every interior ideal of an AG-groupoid $S$ with left identity is a right ideal of $S$.

Theorem 1 Every sI-right-ideal of an AG-groupoid $S$ with left identity is an SI-interiorideal of $S$ over $U$ if and only if $S$ is right duo.

Proof It is simple.

Theorem 2 Let $S$ be a right regular AG-groupoid with left identity. Then, S is left duo if and only if every SI-left-ideal of $S$ over $U$ is an SI-interior-ideal of S over $U$.

Proof Necessity. Let a right regular $S$ with left identity be a left duo, and assume that $f_{A}$ is any si-left-ideal of $S$ over $U$. Let $a, b, c \in S$, then $b \leq x b^{2}$ for some $x \in S$. Since $S a$ is a left ideal of $S$, therefore by hypothesis, $S a$ is a two-sided ideal of $S$. Thus, $a b \cdot c=a(x \cdot b b) \cdot c=$ $a(b \cdot x b) \cdot c=b(a \cdot x b) \cdot c=c(a \cdot x b) \cdot b$. It follows that $a b \cdot c \in S(a \cdot S S) \cdot b \subseteq(S \cdot a S) b=$ $(S S \cdot a S) b=(S a \cdot S S) b \subseteq(S a \cdot S) b \subseteq S a \cdot b$. Thus, $a b \cdot c=t a \cdot b$ for some $t \in S$, and therefore $f_{A}(a b \cdot c)=f_{A}(t a \cdot b) \supseteq f_{A}(b)$, implies that $f_{A}$ is an si-interior-ideal of $S$ over $U$.

Sufficiency. It can be followed from Lemma 8.

By left-right dual of above Theorem, we have the following Theorem:

Theorem 3 Let $S$ be a right regular AG-groupoid with left identity. Then ,S is right duo if and only if every SI-right-ideal of S over $U$ is an SI-interior-ideal of $S$ over $U$.

Lemma 9 A non-empty subset $A$ of a right regular AG-groupoid $S$ with left identity is a two-sided ideal of $S$ if and only if it is an interior ideal of $S$.

Proof It is simple.

Lemma 10 Every left ideal of an AG-groupoid $S$ with left identity is an interior ideal of $S$ if $S$ is an sI-left duo.

Proof It can be followed from Lemmas 1 and 9. 
The converse of Lemma 10 is not true in general. The only left ideal of $S$ (from Example 2) is $\{1,2\}$ which is also an interior ideal of $S$. Let $A=\{2,3,4,5\}$ and define a soft set $f_{A}$ of $S$ over $U=\mathbb{Z}$ as follows:

$$
f_{A}(x)=\left\{\begin{array}{c}
4 \mathbb{Z} \text { if } x=2 \\
8 \mathbb{Z} \text { if } x=3 \\
16 \mathbb{Z} \text { if } x=4 \\
2 \mathbb{Z} \text { if } x=5
\end{array}\right\} .
$$

Then, it is easy to see that $f_{A}$ is an SI -left-ideal of $S$ over $U$ but it is not an SI -right-ideal of $S$ over $U$ because $f_{A}(2 * 4) \nsupseteq f_{A}(2)$.

It is easy to see that every SI-right-ideal of $S$ with left identity over $U$ is an sI-left-ideal of $S$ over $U$.

Remark 5 Every sI-right-ideal of an AG-groupoid S with left identity is an sI-left-ideal of $S$ over $U$, but the converse is not true in general.

Theorem 4 Every right ideal of an AG-groupoid $S$ with left identity is an interior ideal of $S$ if and only if $S$ is an SI-right duo.

Proof It is straightforward.

Theorem 5 Let S be a right regular AG-groupoid with left identity. Then ,S is an SI-left duo if and only if every left ideal of $S$ is an interior ideal of $S$.

Proof The direct part can be followed from Lemma 10. The converse is simple.

By left-right dual of above Theorem, we have the following Theorem.

Theorem 6 Let S be a right regular AG-groupoid with left identity. Then ,S is an SI-right duo if and only if every right ideal of $S$ is an interior ideal of $S$.

Theorem 7 Let $S$ be an AG-groupoid with left identity and $E=\left\{x \in S: x=x^{2}\right\} \subseteq S$. Then the following assertions hold:

(i) Eforms a semilattice;

(ii) $E$ is a singleton set, if $a=a x \cdot a, \forall a, x \in S$.

Proof It is simple.

Theorem 8 For an AG-groupoid $S$ with left identity, the following conditions are equivalent:

(i) $S$ is right regular;

(ii) For any interior ideal I of $S$;

(a) $I \subseteq I^{2}$,

(b) $I$ is semiprime.

(iii) For any SI-interior-ideal $f_{A}$ of $S$ over $U$;

(a) $f_{A} \simeq f_{A} \circ f_{A}$,

(b) $f_{A}$ is soft semiprime over $U$. 
(iv) $S$ is right regular and $|E|=1,(a=a x \cdot a, \forall a, x \in E)$;

(v) $S$ is right regular and $\emptyset \neq E \subseteq S$ is semilattice.

Proof $(i) \Longrightarrow(v) \Longrightarrow(i v)$ can be followed from Theorem 7 .

$(i v) \Longrightarrow($ iii $):(a)$. Let $f_{A}$ be any SI -interior-ideal of a right regular $S$ with left identity. Thus, for each $a \in S$, there exists some $x \in S$ such that $a=x \cdot a a=a \cdot x a=a \cdot x(x \cdot a a)=$ $a \cdot(e x)(a \cdot x a)=a \cdot(x a \cdot a)(x e)$. Therefore,

$$
\begin{aligned}
\left(f_{A} \circ f_{A}\right)(a) & =\bigcup_{a=a \cdot(x a \cdot a)(x e)}\left\{f_{A}(a) \cap f_{A}((x a \cdot a)(x e))\right\} \\
& \supseteq f_{A}(a) \cap f_{A}((x a \cdot a)(x e)) \supseteq f_{A}(a) \cap f_{A}(a)=f_{A}(a) .
\end{aligned}
$$

This shows that $f_{A} \simeq f_{A} \circ f_{A}$.

(b). Also,

$$
\begin{aligned}
a & =x \cdot a a \leq e x \cdot a a=a a \cdot x e=\left(a \cdot x a^{2}\right)(x e)=\left(x \cdot a a^{2}\right)(x e)=x(e a \cdot a a) \cdot(x e) \\
& =x(a a \cdot a e) \cdot(x e)=(a a)(x \cdot a e) \cdot(x e)=(a e \cdot x)(a a) \cdot(x e)=(a e \cdot x) a^{2} \cdot(x e) .
\end{aligned}
$$

This implies that $f_{A}(a)=f_{A}\left((a e \cdot x) a^{2} \cdot(x e)\right) \supseteq f_{A}\left(a^{2}\right)$. Hence, $f_{A}$ is soft semiprime.

(iii) $\Longrightarrow\left(\right.$ ii) : (a). Assume that $I$ is any interior ideal of $S$, then by using Lemma $1, X_{I}$ is an SI-interior-ideal of $S$ over $U$. Let $i \in I$, then by using Lemma 2, we have $X_{I}(i) \subseteq$ $\left(X_{I} \circ X_{I}\right)(i)=\left(X_{I}\right)(i)=U$. Hence, $I \subseteq I^{2}$.

(b). Let $i^{2} \in I$. Then, by given assumption, we have $X_{I}(i) \supseteq X_{I}\left(i^{2}\right)=U$. This implies that $i \in I$, and therefore $I$ is semiprime.

(ii) $\Longrightarrow(i)$ : Let $a \in S$ with left identity. Since $S a^{2}$ is an interior ideals of $S$, and clearly $a^{2} \in S a^{2}$. Thus, by using given assumption, $a \in S a^{2}$. Hence, $S$ is right regular.

Corollary 3 Every SI-interior-ideal of a right regular AG-groupoid S with left identity is soft semiprime over $U$.

Proof Let $f_{I}$ be any SI-interior-ideal of a right regular $S$ with left identity. Then, for each $a \in S$, there exists some $x \in S$ such that $f_{I}(a)=f_{I}(x \cdot a a)=f_{I}(a \cdot x a)=f_{I}\left(x a^{2} \cdot x a\right) \supseteq$ $f_{I}\left(a^{2}\right)$.

Corollary 4 Let I be an interior ideal of an AG-groupoid S. Then, I is semiprime if and only if $X_{I}$ is soft semiprime over $U$.

Theorem 9 Let $S$ be an AG-groupoid with left identity. Then, $S$ is right regular if and only if every SI-interior-ideal $f_{A}$ of $S$ over $U$ is soft idempotent and soft semiprime.

Proof Necessity: Let $f_{A}$ be any si-interior-ideal of a right regular $S$ with left identity over $U$. Then, clearly $f_{A} \circ f_{A} \subseteq f_{A}$. Now for each $a \in S$, there exists some $x \in S$ such that $a=x \cdot a a=a \cdot x a=e a \cdot x a=a x \cdot a e=(a e \cdot x) a$. Thus,

$$
\begin{aligned}
\left(f_{A} \circ f_{A}\right)(a) & =\bigcup_{a=(a e \cdot x) a}\left\{f_{A}(a e \cdot x) \cap f_{A}(a)\right\} \supseteq f_{A}(a e \cdot x) \cap f_{A}(a) \\
& \supseteq f_{A}(a) \cap f_{A}(a)=\left(f_{A} \cap f_{A}\right)(a)=f_{A}(a) .
\end{aligned}
$$

This shows that $f_{A}$ is soft idempotent over $U$. Again $a=e x \cdot a a=a a \cdot x e=a^{2} \cdot x e$. Therefore, $f_{A}(a)=f_{A}\left(a^{2} \cdot x e\right) \supseteq f_{A}\left(a^{2}\right)$. Hence, $f_{A}$ is soft semiprime over $U$. 
Sufficiency: Since $S a^{2}$ is an interior ideal of $S$, therefore by Lemma 1, its soft characteristic function $X_{S a^{2}}$ is an st-interior-ideal of $S$ over $U$ such that $X_{S a^{2}}$ is soft idempotent over $U$. Since by given assumption, $X_{S a^{2}}$ is soft semiprime over $U$ so by Corollary 4,Sa is semiprime. Since $a^{2} \in S a^{2}$, therefore, $a \in a^{2} S$. Thus, by using Lemma 2, we have $X_{S a^{2}} \circ X_{S a^{2}}=X_{S a^{2}}$, and $X_{S a^{2}} \circ X_{S a^{2}}=X_{\left(S a^{2} \cdot S a^{2}\right)}$. Thus, we get $X_{\left(S a^{2} \cdot S a^{2}\right)}=X_{S a^{2}}$. This implies that $X_{\left(S a^{2} \cdot S a^{2}\right)}(a)=X_{S a^{2}}(a)=U$. Therefore, $a \in S a^{2} \cdot S a^{2}=a^{2} S \cdot S a^{2}=\left(S a^{2} \cdot S\right) a^{2} \subseteq S a^{2}$. Hence,$S$ is right regular.

Lemma 11 Every SI-interior-ideal of a right regular AG-groupoid S with left identity over $U$ is soft idempotent.

Proof Let $f_{A}$ be any st-interior-ideal of a right regular $S$ with left identity over $U$. Then, by using Lemma $4, f_{A} \circ f_{A} \simeq f_{A}$. Since $S$ right regular, therefore for every $a \in S$ there exists some $x \in S$ such that $a=x \cdot a a=a \cdot x a=x a^{2} \cdot x a=a x \cdot a^{2} x=\left(a^{2} x \cdot x\right) a=(x x \cdot a a) a=$ $\left(a a \cdot x^{2}\right) a$. Therefore,

$$
\begin{aligned}
& \left(f_{A} \circ f_{A}\right)(a)=\bigcup_{a=\left(a a \cdot x^{2}\right) a}\left\{f_{A}\left(a a \cdot x^{2}\right) \cap f_{A}(a)\right\} \supseteq f_{A}\left(a a \cdot x^{2}\right) \cap f_{A}(a) \\
& \supseteq f_{A}(a) \cap f_{A}(a)=\left(f_{A} \cap f_{A}\right)(a) \text {. }
\end{aligned}
$$

Thus, $f_{A} \circ f_{A}=f_{A}$.

Theorem 10 Let $S$ be an AG-groupoid with left identity and $f_{A}$ be any sI-interior-ideal of $S$ over $U$. Then,$S$ is right regular if and only if $f_{A}=\left(X_{S} \circ f_{A}\right)^{2}$ and $f_{A}$ is soft semiprime.

Proof Necessity: Let $f_{A}$ be any SI-interior-ideal of a right regular $S$ with left identity over $U$. Then, by using Lemmas 4 and 2 , we have

$$
\left(X_{S} \circ\left(X_{S} \circ f_{A}\right)\right) \circ X_{S}=\left(X_{S} \circ f_{A}\right) \circ X_{S}=\left(f_{A} \circ X_{S}\right) \circ X_{S}=\left(X_{S} \circ X_{S}\right) \circ f_{A}=X_{S} \circ f_{A} .
$$

This shows that $X_{S} \circ f_{A}$ is an SI -interior-ideal of $S$ over $U$. Now by using Lemmas 11 and 4, we have $\left(X_{S} \circ f_{A}\right)^{2}=X_{S} \circ f_{A}=f_{A}$. It is easy to see that $f_{A}$ is soft semiprime.

Sufficiency: Let $f_{A}=\left(X_{S} \circ f_{A}\right)^{2}$ holds for any SI-interior-ideal $f_{A}$ of $S$ over $U$. Then, by given assumption and Lemma 14 , we get, $f_{A}=\left(X_{S} \circ f_{A}\right)^{2}=f_{A}^{2}$. Thus, by using Theorem 9, $S$ is right regular.

Corollary 5 Let $S$ be an AG-groupoid with left identity and $f_{A}$ be any st-interiorideal of $S$ over $U$. Then, $S$ is right regular if and only if $f_{A}=X_{S} \circ f_{A}^{2}$ and $f_{A}$ is soft semiprime.

Proof From above theorem, $f_{A}=\left(X_{S} \circ f_{A}\right)^{2}=\left(X_{S} \circ f_{A}\right)\left(X_{S} \circ f_{A}\right)=\left(X_{S} \circ f_{A}\right) \circ f_{A}=$ $\left(f_{A} \circ f_{A}\right) \circ X_{S}=\left(f_{A} \circ f_{A}\right) \circ\left(X_{S} \circ X_{S}\right)=\left(X_{S} \circ X_{S}\right) \circ\left(f_{A} \circ f_{A}\right)=X_{S} \circ f_{A}^{2}$.

Lemma 12 Let $S$ be an AG-groupoid with left identity and $f_{A}$ be any SI-left-ideal (SI right-ideal, sI-two-sided-ideal) of $S$ over $U$. Then, $S$ is right regular if and only if $f_{A}$ is soft idempotent. 
Proof Necessity: Let $f_{A}$ be an si-left-ideal of a right regular $S$ with left identity over $U$. Then, it is easy to see that $f_{A} \circ f_{A} \tilde{\simeq} f_{A}$. Let $a \in S$, then there exists $x \in S$ such that $a=a a \cdot x=x a \cdot a$. Thus

$$
\left(f_{A} \circ f_{A}\right)(a)=\bigcup_{a=x a \cdot a}\left\{f_{A}(x a) \cap f_{A}(a)\right\} \supseteq f_{A}(a) \cap f_{A}(a)=f_{A}(a),
$$

which implies that $f_{A}$ is soft idempotent.

Sufficiency: Assume that $f_{A} \circ f_{A}=f_{A}$ holds for all si-left-ideal of $S$ with a left identity over $U$. Since $S a$ is a left ideal of $S$, therefore by Lemma 1, it follows that $X_{S a}$ is an SI-leftideal of $S$ over $U$. Since $a \in S a$, it follows that $\left(X_{S a}\right)(a)=U$. By hypothesis and Lemma 2, we obtain $\left(X_{S a}\right) \circ\left(X_{S a}\right)=X_{S a}$ and $\left(X_{S a}\right) \circ\left(X_{S a}\right)=X_{S a \cdot S a}$. Thus, we have $\left(X_{S a \cdot S a}\right)(a)=$ $X_{S a}(a)=U$, which implies that $a \in S a \cdot S a$. Therefore, $a \in S a \cdot S a=S^{2} a^{2}=S a^{2}$. This shows that $S$ is right regular.

Theorem 11 Let $S$ be an AG-groupoid with left identity and $f_{A}$ be any sI-left-ideal (SI -right-ideal, sI-two-sided-ideal) of $S$ over $U$. Then, $S$ is right regular if and only if $f_{A}=$ $\left(X_{S} \circ f_{A}\right) \circ\left(X_{S} \circ f_{A}\right)$.

Proof Necessity: Let $S$ be a right regular $S$ with left identity and let $f_{A}$ be any si-left-ideal of $S$ over $U$. It is easy to see that $X_{S} \circ f_{A}$ is also an sI -left-ideal of $S$ over $U$. By Lemma 12, we obtain $\left(X_{S} \circ f_{A}\right) \circ\left(X_{S} \circ f_{A}\right)=\left(X_{S} \circ f_{A}\right) \simeq f_{A}$. Let $a \in S$, then there exists $x \in S$ such that $a=a a \cdot x=x a \cdot a=(x a)(a a \cdot x)=(x a)(x a \cdot a)$. Therefore,

$$
\begin{aligned}
\left(\left(X_{S} \circ f_{A}\right) \circ\left(X_{S} \circ f_{A}\right)\right)(a) & \supseteq\left(X_{S} \circ f_{A}\right)(x a) \cap\left(X_{S} \circ f_{A}\right)(x a \cdot a) \\
& \supseteq X_{S}(x) \cap f_{A}(a) \cap X_{S}(x a) \cap f_{A}(a)=f_{A}(a),
\end{aligned}
$$

which is what we set out to prove.

Sufficiency: Suppose that $f_{A}=\left(X_{S} \circ f_{A}\right) \circ\left(X_{S} \circ f_{A}\right)$ holds for all si-left-ideal $f_{A}$ of $S$ over

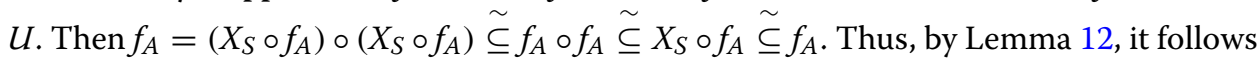
that $S$ is right regular.

Lemma 13 Let $f_{A}$ be any SI-interior-ideal of a right regular AG-groupoid $S$ with left identity over $U$. Then, $f_{A}(a)=f_{A}\left(a^{2}\right)$, for all $a \in S$.

Proof Let $f_{A}$ be any SI-interior-ideal of a right regular $S$ with left identity over $U$. For $a \in S$, there exists some $x$ in $S$ such that $a=e x \cdot a a=a a \cdot x e=(x e \cdot a) a=(x e \cdot a)(e x \cdot a a)=$ $(x e \cdot a)(a a \cdot x e)=a a \cdot(x e \cdot a)(x e)=e a^{2} \cdot(x e \cdot a)(x e)$. Therefore $f_{A}(a)=f_{A}\left(e a^{2} \cdot(x e \cdot a)(x e)\right) \supseteq$ $f_{A}\left(a^{2}\right)=f_{A}(a a)=f_{A}(a(e x \cdot a a))=f_{A}(a(a a \cdot x e))=f_{A}((a a)(a \cdot x e))=f_{A}((x e \cdot a)(a a)) \supseteq$ $f_{A}(a)$. That is, $f_{A}(a)=f_{A}\left(a^{2}\right), \forall a \in S$

The converse of Lemma 13 is not true in general. Let us consider an AG-groupoid $S$ (from Example 2). Let $A=\{1,2,4,5\}$ and define a soft set $f_{A}$ of $S$ over $U=$ $\left\{\left[\begin{array}{ll}0 & 0 \\ x & x\end{array}\right] / x \in \mathbb{Z}_{3}\right\}$ (the set of all $2 \times 2$ matrices with entries from $\mathbb{Z}_{3}$ ) as follows: 


$$
f_{A}(x)=\left\{\begin{array}{c}
\left\{\left[\begin{array}{ll}
0 & 0 \\
0 & 0
\end{array}\right],\left[\begin{array}{ll}
0 & 0 \\
1 & 1
\end{array}\right],\left[\begin{array}{ll}
0 & 0 \\
2 & 2
\end{array}\right]\right\} \text { if } x=1 \\
\left\{\left[\begin{array}{ll}
0 & 0 \\
1 & 1
\end{array}\right],\left[\begin{array}{ll}
0 & 0 \\
2 & 2
\end{array}\right]\right\} \text { if } x=2 \\
\left\{\left[\begin{array}{ll}
0 & 0 \\
2 & 2
\end{array}\right]\right\} \text { if } x=4 \\
\left\{\left[\begin{array}{ll}
0 & 0 \\
1 & 1
\end{array}\right],\left[\begin{array}{ll}
0 & 0 \\
2 & 2
\end{array}\right]\right\} \text { if } x=5
\end{array}\right\} .
$$

It is easy to see that $f_{A}$ is an SI -interior-ideal of $S$ such that $f_{A}(x) \supseteq f_{A}\left(x^{2}\right), \forall x \in S$ but $S$ is not right regular.

On the other hand, it is easy to see that every sI -two-sided-ideal of $S$ over $U$ is an SI -interior-ideal of $S$ over $U$.

Remark 6 Every SI-two-sided-ideal of a right regular AG-groupoid S with left identity over $U$ is an SI-interior-ideal of $S$ over $U$ but the converse is not true in general.

Theorem 12 For an AG-groupoid $S$ with left identity, the following conditions are equivalent:
(i) $S$ is right regular;
(ii) Every interior ideal of $S$ is semiprime;
(iii) Every SI-interior-ideal of $S$ over $U$ is soft semiprime;
(iv) For every SI-interior-ideal $f_{A}$ of $S$ over $U, f_{A}(a)=f_{A}\left(a^{2}\right), \forall a \in S$.

Proof $(i) \Rightarrow$ (iv) can be followed from Lemma 13.

(iv) $\Rightarrow$ (iii) and (iii) $\Rightarrow$ (ii) are obvious.

(ii) $\Rightarrow\left(\right.$ i) : Since $S a^{2}$ is an interior ideal of $S$ with left identity such that $a^{2} \in S a^{2}$, therefore by given assumption, we have $a \in S a^{2}$. Thus, $S$ is right regular.

\section{Weakly regular $A G^{* * *}$-groupoids}

An AG-groupoid $S$ is called an $A G^{* * *}$-groupoid [29] if the following conditions are satisfied:

(i) For all $a, b, c \in S, a \cdot b c=b \cdot a c$;

(ii) For all $a \in S$, there exist some $b, c \in S$ such that $a=b c$.

An AG-groupoid satisfying (i) is called an $A G^{* * *}$-groupoid. The condition (ii) for an $\mathrm{AG}^{* * *}$-groupoid to become an $\mathrm{AG}^{* * * *}$-groupoid is equivalent to $S=S^{2}$.

Let $S=\{1,2,3,4\}$ be an AG-groupoid define in the following multiplication table.

\begin{tabular}{l|llll}
$\cdot$ & 1 & 2 & 3 & 4 \\
\hline 1 & 1 & 1 & 1 & 1 \\
\hline 2 & 1 & 4 & 3 & 4 \\
\hline 3 & 1 & 2 & 4 & 4 \\
\hline 4 & 1 & 4 & 4 & 4 \\
\hline
\end{tabular}

It is easy to verify that $(S, \cdot)$ is an $\mathrm{AG}^{\text {**** }}$-groupoid.

Note that every AG-groupoid with left identity is an $\mathrm{AG}^{* * *}$-groupoid but the converse is not true in general. An AG-groupoid in the above example is an $\mathrm{AG}^{* * * *}$-groupoid, but it does not contains a left identity. Hence, we can 
say that an $\mathrm{AG}^{\text {*w* }}$-groupoid is the generalization of an AG-groupoid with left identity.

An element $a$ of an AG-groupoid $S$ is called a weakly regular element of $S$ if there exist some $x, y \in S$ such that $a=a x \cdot a y$ and $S$ is called weakly regular if every element of $S$ is weakly regular.

Remark 7 Let $S$ be an $A G^{* * *}$-groupoid. Then, the concepts of weak and right regularity coincide in $S$.

Let $S$ be an $\mathrm{AG}^{* * * *}$-groupoid. From now onward, $R$ (resp. $L$ ) will denote any right (resp. left) ideal of $S$; $\langle R\rangle_{a^{2}}$ will denote a right ideal $S a^{2} \cup a^{2}$ of $S$ containing $a^{2}$ and $\langle L\rangle_{a}$ will denote a left ideal $S a \cup a$ of $S$ containing $a$; $f_{A}$ (resp. $g_{B}$ ) will denote any si-right-ideal of $S$ (resp. si-left-ideal of $S$ ) over $U$ unless otherwise specified.

Theorem 13 Let $S$ be an $A G^{* * * *}$-groupoid. Then, $S$ is weakly regular if and only if $\langle R\rangle_{a^{2}} \cap$ $\langle L\rangle_{a}=\langle R\rangle_{a^{2}}^{2}\langle L\rangle_{a}^{2}$ and $\langle R\rangle_{a^{2}}$ is semiprime.

Proof Necessity: Let $S$ be weakly regular. It is easy to see that $\langle R\rangle_{a^{2}}^{2}\langle L\rangle_{a}^{2} \subseteq\langle R\rangle_{a^{2}} \cap\langle L\rangle_{a}$. Let $a \in\langle R\rangle_{a^{2}} \cap\langle L\rangle_{a}$. Then, there exist some $x, y \in S$ such that

$$
\begin{aligned}
a & =a x \cdot a y=(a x \cdot a y) x \cdot(a x \cdot a y) y=(x \cdot a y)(a x) \cdot(y \cdot a y)(a x) \\
& =(a \cdot x y)(a x) \cdot\left(a y^{2}\right)(a x)=(a \cdot x y)(a x) \cdot(x a)\left(y^{2} a\right) \\
& \in\left(\langle R\rangle_{a^{2}} S \cdot\langle R\rangle_{a^{2}} S\right)\left(S\langle L\rangle_{a} \cdot S\langle L\rangle_{a}\right) \subseteq\langle R\rangle_{a^{2}}^{2}\langle L\rangle_{a}^{2},
\end{aligned}
$$

which shows that $\langle R\rangle_{a^{2}} \cap\langle L\rangle_{a}=\langle R\rangle_{a^{2}}^{2}\langle L\rangle_{a}^{2}$. It is easy to see that $\langle R\rangle_{a^{2}}$ is semiprime.

Sufficiency: Since $S a^{2} \cup a^{2}$ and $S a \cup a$ are the right and left ideals of $S$ containing $a^{2}$ and $a$ respectively. Thus, by using given assumption, we get

$$
\begin{aligned}
a & \in\left(S a^{2} \cup a^{2}\right) \cap(S a \cup a)=\left(S a^{2} \cup a^{2}\right)^{2}(S a \cup a)^{2} \\
& =\left(S a^{2} \cup a^{2}\right)\left(S a^{2} \cup a\right) \cdot(S a \cup a)(S a \cup a) \\
& \subseteq S\left(S a^{2} \cup a\right) \cdot S(S a \cup a)=\left(S \cdot S a^{2} \cup S a\right)(S \cdot S a \cup S a) \\
& =\left(a^{2} S \cdot S \cup S a\right)(a S \cdot S \cup S a)=\left(S a^{2} \cup S a\right)(S a \cup S a) \\
& =\left(a^{2} S \cup S a\right)(S a \cup S a)=(S a \cdot a \cup S a)(S a \cup S a) \\
& \subseteq(S a \cup S a)(S a \cup S a)=S a \cdot S a=a S \cdot a S .
\end{aligned}
$$

This implies that $S$ is weakly regular.

Corollary 6 Let $S$ be an $A G^{* * *}$-groupoid. Then, $S$ is weakly regular if and only if $\langle R\rangle_{a^{2}} \cap$ $\langle L\rangle_{a}=\langle L\rangle_{a}^{2}\langle R\rangle_{a^{2}}^{2}$ and $\langle R\rangle_{a^{2}}$ is semiprime.

Theorem 14 Let $S$ be an $A G^{* * * *}$-groupoid. Then, the following conditions are equivalent:

(i) $S$ is weakly regular;

(ii) $\langle R\rangle_{a^{2}} \cap\langle L\rangle_{a}=\langle L\rangle_{a}^{2}\langle R\rangle_{a^{2}}^{2}$ and $\langle R\rangle_{a^{2}}$ is semiprime;

(iii) $R \cap L=L^{2} R^{2}$ and $R$ semiprime;

(iv) $f_{A} \cap g_{B}=\left(f_{A} \circ g_{B}\right) \circ\left(f_{A} \circ g_{B}\right)$ and $f_{A}$ is soft semiprime;

(v) $S$ is weakly regular and $|E|=1,(a=a x \cdot a, \forall a, x \in E)$;

(vi) $S$ is weakly regular and $\emptyset \neq E \subseteq S$ is semilattice. 
Proof $(i) \Longrightarrow(v i) \Longrightarrow(v)$ : It can be followed from Theorem 7 .

$(v) \Longrightarrow(i v):$ Let $f_{A}$ and $g_{B}$ be any sI -right-ideal and si-left-ideal of a weakly regular $S$ over $U$ respectively. From Lemma 5, it is easy to show that $\left(f_{A} \circ g_{B}\right) \circ\left(f_{A} \circ g_{B}\right) \widetilde{\simeq} f_{A} \tilde{\cap} g_{B}$. Now for $a \in S$, there exist some $x, y \in S$ such that

$$
\begin{aligned}
a & =a x \cdot a y=(a x \cdot a y) x \cdot(a x \cdot a y) y=(a x \cdot a y) \cdot((a x \cdot a y) x) y \\
& =(a x \cdot a y) \cdot(y x)(a x \cdot a y)=(a x \cdot a y) \cdot(a x)(y x \cdot a y) \\
& =(a x \cdot a y) \cdot(a y \cdot y x)(x a)=(a x \cdot a y) \cdot((y x \cdot y) a)(x a) \\
& =(a x)((y x \cdot y) a) \cdot(a y)(x a)=(a x)(b a) \cdot(a y)(x a), \text { where } y x \cdot y=b .
\end{aligned}
$$

Therefore,

$$
\begin{aligned}
\left(\left(f_{A} \circ g_{B}\right) \circ\left(f_{A} \circ g_{B}\right)\right)(a)= & \bigcup_{a=(a x)(b a) \cdot(a y)(x a)}\left\{\left(f_{A} \circ g_{B}\right)(a x \cdot b a)\right. \\
& \left.\supseteq\left(f_{A} \circ g_{B}\right)(a y \cdot x a)\right\} \\
& \bigcup_{a x \cdot b a=a x \cdot b a}\left\{f_{A}(a x) \cap g_{B}(b a)\right\} \\
& \cap \bigcup_{a y \cdot x a=a y \cdot x a}\left\{f_{A}(a y) \cap g_{B}(x a)\right\} \\
& \supseteq f_{A}(a x) \cap g_{B}(b a) \cap f_{A}(a y) \cap g_{B}(x a) \\
& \supseteq f_{A}(a) \cap g_{B}(a),
\end{aligned}
$$

which shows that $\left(f_{A} \circ g_{B}\right) \circ\left(f_{A} \circ g_{B}\right) \supseteq f_{A} \tilde{\cap} g_{B}$. Hence, $f_{A} \tilde{\cap} g_{B}=\left(f_{A} \circ g_{B}\right) \circ\left(f_{A} \circ g_{B}\right)$. Also by using Lemma $3, f_{A}$ is soft semiprime.

(iv) $\Longrightarrow$ (iii) : Let $R$ and $L$ be any left and right ideals of $S$. Then, by using Lemma 1, $X_{R}$ and $X_{L}$ are the si-right-ideal and si-left-ideal of $S$ over $U$ respectively. Now by using Lemma 2, we get $X_{R \cap L}=X_{R} \tilde{\cap} X_{L}=\left(X_{R} \circ X_{L}\right) \circ\left(X_{R} \circ X_{L}\right)=\left(X_{R} \circ X_{R}\right) \circ\left(X_{L} \circ X_{L}\right)=$ $X_{R^{2}} \circ X_{L^{2}}=X_{R^{2} L^{2}}=X_{L^{2} R^{2}}$, which implies that $R \cap L=L^{2} R^{2}$.

(iii) $\Longrightarrow$ (ii) : It is simple.

(ii) $\Longrightarrow($ i $)$ : It can be followed from Corollary 6 .

Lemma 14 Let $R$ be a right ideal and $L$ be a left ideal of a unitary AG-groupoid $S$ with left identity respectively. Then, $R L$ is a left ideal of $S$.

Proof It is simple.

Theorem 15 Let $S$ be an $A G^{* * * *}$-groupoid. Then, the following conditions are equivalent:

(i) $S$ is weakly regular;

(ii) $\langle R\rangle_{a^{2}} \cap\langle L\rangle_{a}=\langle R\rangle_{a^{2}}\langle L\rangle_{a} \cdot\langle R\rangle_{a^{2}}$ and $\langle R\rangle_{a^{2}}$ is semiprime;

(iii) $R \cap L=R L \cdot R$ and $R$ is semiprime;

(iv) $f_{A} \tilde{\cap} g_{B}=\left(f_{A} \circ g_{B}\right) \circ f_{A}$ and $f_{A}$ is soft semiprime;

(v) $S$ is weakly regular and $|E|=1,(a=a x \cdot a, \forall a, x \in E)$;

(vi) $S$ is weakly regular and $\emptyset \neq E \subseteq S$ is semilattice.

Proof $(i) \Longrightarrow(v i) \Longrightarrow(v)$ : It can be followed from Theorem 7 . 
$(v) \Longrightarrow(i v)$ : Let $f_{A}$ and $g_{B}$ be any SI -left-ideals of a weakly regular $S$ over $U$. Now, for $a \in S$, there exist some $x, y \in S$ such that $a=a x \cdot a y=a x \cdot(a x \cdot a y) y=((a x \cdot a y) y \cdot x) a=$ $(x y \cdot(a x \cdot a y)) a=(a x \cdot(x y \cdot a y)) a=(a x \cdot(a \cdot(x y) y)) a$.

Therefore,

$$
\begin{aligned}
\left(\left(f_{A} \circ g_{B}\right) \circ f_{A}\right)(a) & =\bigcup_{a=(a x \cdot(a \cdot(x y) y)) a}\left\{\left(f_{A} \circ g_{B}\right)(a x \cdot(a \cdot(x y) y)) \cap g_{B}(a)\right\} \\
& \supseteq \bigcup_{a x \cdot(a \cdot(x y) y=a x \cdot(a \cdot(x y) y}\left\{f_{A}(a x) \cap g_{B}(a \cdot(x y) y)\right\} \cap g_{B}(a) \\
& \supseteq f_{A}(a x) \cap g_{B}(a \cdot(x y) y) \cap g_{B}(a) \supseteq f_{A}(a) \cap g_{B}(a),
\end{aligned}
$$

which shows that $\left(f_{A} \circ g_{B}\right) \circ f_{A} \supseteq f_{A} \tilde{\cap} g_{B}$. By using Lemmas 5 and 3 , it is easy to show that $\left(f_{A} \circ g_{B}\right) \circ f_{A} \subseteq f_{A} \sim{ }^{\sim} g_{B}$. Thus, $f_{A} \sim g_{B}=\left(f_{A} \circ g_{B}\right) \circ f_{A}$. Also, by using Lemma $3, f_{A}$ is soft semiprime.

(iv) $\Longrightarrow$ (iii) : Let $R$ and $L$ be any left and right ideals of $S$ respectively. Then, by Lemma $1, X_{R}$ and $X_{L}$ are the sI-right-ideal and SI -left-ideal of $S$ over $U$ respectively. Now, by using Lemmas 2,14 , we get $X_{R \cap L}=X_{R} \tilde{\sim} X_{L}=\left(X_{R} \circ X_{L}\right) \circ X_{L}=X_{R L \cdot R}$, which shows that $R \cap L=R L \cdot R$. Also, by using Lemma $6, R$ is semiprime.

(iii) $\Longrightarrow($ ii) : It is obvious.

(ii) $\Longrightarrow(i)$ : Since $S a^{2} \cup a^{2}$ and $S a \cup a$ are the right and left ideals of $S$ containing $a^{2}$ and $a$ respectively. Thus, by using given assumption and Lemma, we get

$$
\begin{aligned}
a & \in\left(S a^{2} \cup a^{2}\right) \cap(S a \cup a)=\left(S a^{2} \cup a^{2}\right)(S a \cup a) \cdot\left(S a^{2} \cup a^{2}\right) \\
& \subseteq S(S a \cup a) \cdot\left(S a^{2} \cup a^{2}\right)=\left(S^{2} a \cup S a\right)\left(S a^{2} \cup a^{2}\right) \\
& =\left(S^{2} a \cdot S a^{2}\right) \cup\left(S^{2} a \cdot a^{2}\right) \cup\left(S a \cdot S a^{2}\right) \cup\left(S^{2} a \cdot a^{2}\right) \\
& \subseteq\left(S a \cdot a^{2} S\right) \cup(S a \cdot S a) \cup\left(S a \cdot a^{2} S\right) \cup(S a \cdot S a) \\
& \subseteq(S a \cdot S a) \cup(S a \cdot S a) \cup(S a \cdot S a) \cup(S a \cdot S a) \\
& =S a \cdot S a=a S \cdot a S .
\end{aligned}
$$

Hence, $S$ is weakly regular.

\section{Comparison of SI-left (right, two-sided, interior) ideals}

A very major and an abstract conclusion from this section is that sI-left-ideal, sI-rightideal and SI-interior-ideal need not to be coincide in an AG-groupoid $S$ even if $S$ has a left identity, but they will coincide in a right regular class of an AG-groupoid $S$ with left identity.

E-1. Take a collection of 8 chemicals as an initial universe set $U$ given by $U=$ $\left\{s_{1}, s_{2}, s_{3}, s_{4}, s_{5}, s_{6}, s_{7}, s_{8}\right\}$.

Let a set of parameters $S=\{1,2,3,4,5\}$ be a set of particular properties of each chemical in $U$ with the following type of natures:

1 stands for the parameter "density",

2 stands for the parameter "melting point",

3 stands for the parameter "combustion",

4 stands for the parameter "enthalpy",

5 stands for the parameter "toxicity". 
Let us define the following binary operation on a set of parameters $S$ as follows.

\begin{tabular}{l|lllll}
$*$ & 1 & 2 & 3 & 4 & 5 \\
\hline 1 & 1 & 1 & 1 & 1 & 1 \\
2 & 1 & 2 & 2 & 2 & 2 \\
3 & 1 & 2 & 4 & 5 & 3 \\
4 & 1 & 2 & 3 & 4 & 5 \\
5 & 1 & 2 & 5 & 3 & 4
\end{tabular}

It is easy to check that $(S, *)$ is non-commutative and non-associative. Also, by routine calculation, one can easily verify that $(S, *)$ forms an AG-groupoid with left identity 4 . Note that $S$ is left (right) regular. Indeed, for $a \in S$ there does exists some $x \in S$ such that $a=x a^{2}\left(a=a^{2} x\right)$.

Let $A=S$ and define a soft set $f_{A}$ of $S$ over $U$ as follows:

$f_{A}(x)=\left\{\begin{array}{c}\left\{s_{1}, s_{2}, s_{3}, s_{4}, s_{5}, s_{6}\right\} \text { if } x=1 \\ \left\{s_{2}, s_{3}, s_{4}\right\} \text { if } x=2 \\ \left\{s_{2}, s_{3} \text { if } x=3=4=5\right.\end{array}\right\}$.

Then, it is easy to verify that $f_{A}$ is an SI -interior-ideal of $S$ over $U$.

E-2. There are seven civil engineers in an initial universe set $U$ given by $U=$ $\left\{s_{1}, s_{2}, s_{3}, s_{4}, s_{5}, s_{6}, s_{7}\right\}$.

Let a set of parameters $S=\{1,2,3\}$ be a set of status of each civil engineer in $U$ with the following type of attributes:

1 stands for the parameter "critical thinking",

2 stands for the parameter "decision making",

3 stands for the parameter "project management".

Let us define the following binary operation on a set of parameters $S$ as follows.

\begin{tabular}{l|lll}
$*$ & 1 & 2 & 3 \\
\hline 1 & 1 & 1 & 1 \\
2 & 3 & 3 & 3 \\
3 & 1 & 1 & 1 \\
\hline
\end{tabular}

It is easy to check that $(S, *)$ is non-commutative and non-associative. One can easily verify that $(S, *)$ forms an AG-groupoid. Note that $S$ is not left (right) regular. Indeed for $3 \in S$ there does not exists some $x \in S$ such that $3=x * 3^{2}\left(3=3^{2} * x\right)$. Let $A=S$ and define a soft set $f_{A}$ of $S$ over $U$ as follows:

$$
f_{A}(x)=\left\{\begin{array}{c}
\left\{s_{1}, s_{2}, s_{3}, s_{4}\right\} \text { if } x=1 \\
\left\{s_{1}, s_{2}, s_{3}\right\} \text { if } x=2 \\
\left\{s_{2}, s_{3}\right\} \text { if } x=3
\end{array}\right\} .
$$

Then, it is easy to verify that $f_{A}$ is an SI -interior-ideal of $S$ over $U$ but it is not an SI -left-ideal, sI-right-ideal, and SI -interior-ideal of $S$ which can be seen from the following:

$$
f_{A}(2 * 2) \nsupseteq f_{A}(2) \text { and } f_{A}(3 * 2) \nsupseteq f(2) \text {. }
$$

Lemma 15 Every SI-right-ideal of an AG-groupoid S with left identity over U is an SIleft-ideal of $S$ over $U$.

Proof It is simple.

The converse of above Lemma is not true in general which can be seen from the following example. 
E-3. Let us consider an AG-groupoid $S$ with left identity 4 given in an Example 1 with an initial universe set $U=\left\{s_{1}, s_{2}, \ldots, s_{12}\right\}$. Let a set of parameters $S=\{1,2,3,4,5\}$ be a set of status of houses in which,

1 stands for the parameter "beautiful",

2 stands for the parameter "cheap",

3 stands for the parameter "in good location",

4 stands for the parameter "in green surroundings",

5 stands for the parameter "secure".

It is important to note that $S$ is not right regular because for $3 \in S$ there does not exists some $x \in S$ such that $3=x * 3^{2}$.

Let $A=S$ and define a soft set $f_{A}$ of $S$ over $U$ as follows:

$$
f_{A}(x)=\left\{\begin{array}{c}
U \text { if } x=1 \\
\left\{s_{2}, s_{3}, s_{4}, s_{5}, s_{6}, s_{7}, s_{8}\right\} \text { if } x=2 \\
\left\{s_{2}, s_{3}, s_{4}, s_{5}, s_{6}\right\} \text { if } x=3 \\
\left\{s_{2}, s_{3}, s_{4}, s_{5}\right\} \text { if } x=4 \\
\left\{s_{1}, s_{2}, s_{3}, s_{4}, s_{5}, s_{6}, s_{7}, s_{8}, s_{9}, s_{10}\right\} \text { if } x=5
\end{array}\right\} .
$$

It is easy to verify that $f_{A}$ is an si-left-ideal of $S$ over $U$, but it is not an si-right-ideal of $S$ over $U$, because $f_{A}(2 * 4) \nsupseteq f_{A}(2)$. Also, one can easily see that $f_{A}$ is an sI-interior-ideal of $S$ over $U$ but it is not an sI-two-sided-ideal of $S$ over $U$.

Note that every SI-two-sided-ideal of an AG-groupoid $S$ with left identity over $U$ is an sI-interior-ideal of $S$ over $U$.

Theorem 16 Let $f_{A}$ be any soft set of a right regular AG-groupoid $S$ with left identity over $U$. Then, $f_{A}$ is an SI-left-ideal of $S$ over $U$ if and only if $f_{A}$ is an SI-right-ideal of $S$ over $U$ if and only if $f_{A}$ is an SI-two-sided-ideal of $S$ over $U$ if and only if $f_{A}$ is an SI-interior-ideal of $S$ over $U$.

Proof Assume that $f_{A}$ is any si-left-ideal of a right regular $S$ with left identity over $U$. Let $a, b \in S$. For $a \in S$, there exists some $x \in S$ such that $a=x a^{2}$. Thus, $a b=x a^{2}$. $b=(a \cdot x a) b=(b \cdot x a) a$. Therefore, $f_{A}((b \cdot x a) a) \supseteq f_{A}(a)$. Now, by using Lemma 15 , $f_{A}$ is an si-left-ideal of $S$ over $U$ if and only if $f_{A}$ is an si-right-ideal of $S$ over $U$. Let $f_{A}$ is any si-right-ideal of a right regular with left identity over $U$. Let $a, b, c \in S$, then $f_{A}(a b \cdot c)=f_{A}\left(\left(x a^{2} \cdot b\right) c\right)=f_{A}\left(c b \cdot x a^{2}\right)=f_{A}\left(a^{2} x \cdot b c\right)=f_{A}\left(b\left(a^{2} x \cdot c\right)\right) \supseteq f_{A}(b)$. Again assume that $f_{A}$ is any SI -interior-ideal of a right regular $S$ with left identity over $U$. Thus, $f_{A}(a b) \supseteq f_{A}\left(x a^{2} \cdot b\right) \supseteq f_{A}\left(a^{2}\right)=f_{A}\left(x a^{2} \cdot x a^{2}\right)=f_{A}\left(a^{2} x \cdot a^{2} x\right)=f_{A}\left((a a)\left(a^{2} x \cdot x\right)\right) \supseteq f_{A}(a)$, which is what we set out to prove.

\section{Conclusions}

Every AG-groupoid with left identity can be considered as an $\mathrm{AG}^{* * * *}$-groupoid, but the converse is not true in general. This leads us to the fact that an $A G^{* * * *}$-groupoid can be seen as the generalization of an AG-groupoid with left identity. Thus, the results of "Right regular AG-groupoids" section can be trivially followed for an $\mathrm{AG}^{* * * *}$ groupoid.

The idea of soft sets in an AG-groupoid will help us in verifying the existing characterizations and to achieving new and generalized results in future works. Some of them are as under: 
1. To generalize the results of a semigroups using soft sets.

2. To characterize a newly developed substructure called an $A^{* * * * *}$-groupoid through soft sets.

3. To study the structural properties of an AG-hypergroupoid by using soft sets.

4. To introduce and examine the concept of a $\Gamma$-AG-groupoid in terms of soft sets.

Acknowledgements

The authors are grateful to the reviewers' valuable comments that improved the manuscript.

\section{Authors' contributions}

Both authors contributed equally. Both authors read and approved the final manuscript.

Funding

No any place give us supporting or funding, only by the author.

\section{Availability of data and materials}

No data were used to support this study.

\section{Competing interests}

The authors declare that they have no competing interests.

\section{Author details}

${ }^{1}$ Military College of Engineering, National University of Sciences and Technology (NUST), Islamabad, Pakistan. ${ }^{2}$ Higher Institute of Engineering and Technology, King Mariout, Alexandria, Egypt.

Received: 3 April 2019 Accepted: 2 December 2019

Published online: 30 December 2019

\section{References}

1. Ali, M. I., Feng, F., Liu, X., Mine, W. K., Shabir, M: On some new operations in soft set theory, Comput. Math. Appl. 57, 1547-1553 (2009)

2. Cagman, N., Enginoglu, S.: FP-soft set theory and its applications. Ann. Fuzzy Math. Inform. 2, 219-226 (2011)

3. Dudek, W. A., Gigon, R. S.: Congruences on completely inverse AG**-groupoids. Quasigroups Relat. Syst. 20, 203-209 (2012)

4. Dudek, W. A., Gigon, R. S.: Completely inverse AG**-groupoids. Semigroup Forum. 87, 201-229 (2013)

5. Feng, F.: Soft rough sets applied to multicriteria group decision making. Ann. Fuzzy Math. Inform. 2, 69-80 (2011)

6. Feng, F., Jun, Y. B., Zhao, X.: Soft semirings. Comput. Math. Appl. 56, 2621-2628 (2008)

7. Jun, Y. B.: Soft BCK/BCl-algebras, Comput. Math. Appl. 56, 1408-1413 (2008)

8. Jun, Y. B., Ahn, S. S.: Double-framed soft sets with applications in BCK/BCl-algebras (2012)

9. Jun, Y. B., Lee, K. J., Khan, A.: Soft ordered semigroups. Math. Logic Q. 56, 42-50 (2010)

10. Kazim, M. A., Naseeruddin, M.: On almost semigroups. The Alig. Bull. Math. 2, 1-7 (1972)

11. Khan, M.: Some studies in AG $^{*}$-groupoids, Ph. D Thesis. Quaid-i-Azam University, Pakistan (2008)

12. Kovkov, D. V., Kolbanov, V. M., Molodtsov, D. A.: Soft sets theory based optimization. J. Comput. Syst. Sci. Int. 46(6), 872-880 (2007)

13. Maji, P. K., Roy, A. R., Biswas, R.: an application of soft sets in a decision making problem, Comput. Math. Appl. 44, 1077-1083 (2002)

14. Molodtsov, D., Leonov, V. Y., Kovkov, D. V.: Soft sets technique and its application. Nechetkie Sistemy i Myagkie Vychisleniya. 1(1), 8-39 (2006)

15. Majumdar, P., Samanta, S. K.: Similarity measures of soft sets. New Math. Neutral Comput. 4(1), 1-12 (2008)

16. Molodtsov, D.: Soft set theory. Comput. Math. Appl. 37, 19-31 (1999)

17. Mushtaq, Q., Yusuf, S. M: On LA-semigroups. The Alig. Bull. Math. 8, 65-70 (1978)

18. Mushtaq, Q., Yusuf, S. M: On locally associative left almost semigroups. J. Nat. Sci. Math. 19, 57-62 (1979)

19. Pei, D. W., Miao, D.: From soft sets to information systems. IEEE Int. Conf. Granul. Comput., 617-621 (2005)

20. Protić P.V., Stevanović, N.: AG-test and some general properties of Abel-Grassmann's groupoids. PU. M. A. 4(6), 371-383 (1995)

21. Roy, A. R., Maji, P. K.: A fuzzy soft set theoretic approach to decision making problems. J. Comput. Appl. Math. 203 412-418 (2007)

22. Sezgin, A., Atagun, A. O., Cagman, N.: Soft intersection nearrings with applications. Neural Comput. Appl. 21, 221-229 (2011)

23. Seizgin, A., Atagun, A. O.: On operations of soft sets, Comput. Math. Appl. 61, 1457-1467 (2011)

24. Sezgin, A.: A new approach to LA-semigroup theory via the soft sets. J. Intell. Fuzzy Syst. 26, 2483-2495 (2014)

25. Shah, T., Razzaque, A., Rehman, I.: Application of soft sets to non-associative rings. J. Intell. Fuzzy Syst. 30, 1537-1564 (2016)

26. Shah, T, Razzaque, A: Soft M-systems in a class of soft non-associative rings. U. P. B. Sci. Bull. Ser. A. 77, 131-142 (2015)

27. Stevanović N., Protić P.V.: Composition of Abel-Grassmann's 3-bands. Novi Sad. Math. J. 2, 175-182 (2004)

28. Yang, X., Yu, D., Yang, J., Wu, C.: Generalization of soft set theory from crisp to fuzzy case. Fuzzy Inormation Eng. 40 345-355 (2007) 
29. Yousafzai, F., Ali, A., Haq, S., Hila, K.: Non-associative semigroups in terms of semilattices via soft ideals. J. Intell. Fuzzy Syst. 35, 4837-4847 (2018)

\section{Publisher's Note}

Springer Nature remains neutral with regard to jurisdictional claims in published maps and institutional affiliations.

Submit your manuscript to a SpringerOpen ${ }^{\circ}$ journal and benefit from:

- Convenient online submission

- Rigorous peer review

- Open access: articles freely available online

- High visibility within the field

- Retaining the copyright to your article

Submit your next manuscript at $\gg$ springeropen.com 